\title{
Reasons for use and non-use of dental services among people visiting a dental college hospital in India: A descriptive cross-sectional study
}

CG Devaraj

Pranati Eswar ${ }^{2}$

\begin{abstract}
Objective: To find out the reasons for use and non-use of dental services among people visiting a dental college hospital in India.

Methods: A descriptive cross-sectional study was carried out on 180 people, aged 15-65 years visiting the outpatient department of a dental college hospital in India. A self-administered questionnaire was used to collect data about the history of dental visits and reasons for use and non-use of dental services.

Results: For a majority of the people (38.5\%), time since the last dental visit was more than 2 years, and the main reason for visiting a dentist was dental pain (35.3\%). Majority of the people had undergone extraction of teeth in their previous dental visit (44.3\%). The major reason cited for not visiting a dentist was the presence of dental problem that was not severe enough to go to a dentist (43.1\%).
\end{abstract}

Conclusion: Majority of the people were "problem-oriented visitors" rather than "prevention oriented visitors." (Eur J Dent 2012;6:422-427)

Key words: Dental services; utilization; barriers; dental college hospital; India

1 Department of Periodontology and Implanatology, Mahatma Gandhi Dental College and Hospital, RIICO Institutional area, Sitapura, Jaipur -302002, Rajasthan, INDIA

2 Department of Public Health Dentistry, Mahatma Gandhi Dental College and Hospital, RIICO Institutional area,

- Sitapura, Jaipur -302002, Rajasthan, INDIA

Corresponding author: Dr. CG Devaraj

Department of Periodontology and Implantology, Mahatma Gandhi Dental College and Hospital, RIICO Institutional area, Sitapura, Jaipur -302022, Rajasthan, INDIA

Tel: +919783515551

Fax: +911412770326

Email: dr_devarajcglyahoo.com

\section{INTRODUCTION}

Oral diseases like dental caries, periodontitis, and oral cancer are major public health problems in high-income countries, and the burden of oral diseases is growing in many low- and middle-income countries. ' These diseases are generally not self-limiting, and untreated problems often negatively affect a person's well-being and general quality of life. ${ }^{2}$ However, oral diseases are largely preventable by regular home oral care and preventive dental visits. ${ }^{3}$ 
Preventive dental visits help in the early detection and treatment of oral diseases. But the provision of preventive dental care for adults depends on each patient's initiatives in utilizing dental care. ${ }^{4}$ Dental service use can be defined by the following parameters: (1) annual number of dental visits per person, (2) proportion of persons visiting a dentist within a year, (3) reported first dental visit within a series of visits, (4) lack of dental visits within a specific period of time, (5) aggregated expenditures for dental visits, and (6) routine vs. emergency care. ${ }^{5}$

Dental care use is influenced by a complex set of factors. Behavioral, socioeconomic, and culturally related predisposing enabling and need-based factors contribute to people's decisions to either forgo care or seek professional assistance for dental problems. ${ }^{6,7,8,9}$ To improve oral health outcomes, an adequate knowledge of the way individuals use health services and the factors predictive of this behavior is essential. ${ }^{10}$

In many developing countries, dental care utilization is limited, and teeth are often left untreated or extracted. ${ }^{10}$ Though dentists recommend regular dental visits, many people fail to comply with this due to several barriers that exist for the utilization of dental services. The barriers for seeking dental services have been classified by the FDI as related to the following: (a) individuals themselves (such as the lack of perceived need, anxiety or fear, financial considerations, and lack of access), (b) dental profession linappropriate manpower resources, uneven geographical distribution, training inappropriate to changing needs and demands, and insufficient sensitivity to patient's attitudes and needs), and (c) society (insufficient public support of attitudes conducive to health, inadequate oral health care facilities, inadequate oral health manpower planning, and insufficient support for research). ${ }^{11}$

Understanding factors that initiate and hinder dental service use is necessary if decision makers are to develop policies that adequately address these issues. In this direction, utilization studies serve as an important tool for oral health policy decision making and understanding oral healthrelated behavior.

The search of available literature revealed a relative scarcity of Indian studies on the dental treatment-seeking behavior of people. Hence, the present study was conducted with an objective of finding out the reasons for use or non-use of dental services among the people visiting a dental college hospital in India.

\section{MATERIALS AND METHODS}

The study was reviewed by the institutional review board, and clearance was obtained. A voluntary, written informed consent was obtained from the participants before the start of the study.

The time limit set for collection of data was for a period of 5 continuous working days of the dental college hospital. The study was carried out in the outpatient department of the hospital. The sampling methodology adopted was deliberate sampling. The study sample comprised of all patients visiting the outpatient department of the hospital during the 5-day period.

The study sample consisted of 180 people, aged 15-65 years. Out of the 180 people, 122 people had previously visited a dentist, and 58 people had never previously visited a dentist.

A self-administered, anonymous questionnaire prepared in the local language was used to collect data about socio-demographic characteristics, dental visit history, and reasons for not visiting a dentist. The internal consistency of the questionnaire was pilot tested on 50 subjects, and Cronbach's alpha value of 0.87 was obtained.

The people who had previously visited a dentist were asked to report the time since their last dental visit, reason for their last dental visit, and the treatment received during their last dental visit.

On oral examination by the author, it was found that all the 58 people who had never previously visited a dentist had dental problems that required treatment. Out of the 58 people who had never previously visited a dentist, 26 people had a deep cavity in at least one of their teeth, 14 people had 1 or more missing teeth, 11 people had stains and deposits on their teeth, and 7 people had mobility in 1 or more teeth. These people were asked to choose from a list, the reason that had prevented them from converting their need for treatment into demand for dental care. A space was also provided for listing reasons not included in the choices. 
The data was systematically compiled, and the results were expressed in percentages.

\section{RESULTS}

The study results showed that majority of people who had previously visited a dentist belonged to the age group of $15-25$ years (31\%), and majority of people who had never previously visited a dentist belonged to the age group of 26-35 years (40\%).

More number of females had visited a dentist previously (56\%) as compared to males (47\%).

When the place of residence was taken into consideration, the urban people had visited a dentist more $(66 \%)$ than the rural people did (50\%).

Among the people who had previously visited a dentist, a higher percentage of people had highschool level of education (37\%), followed by college level of education (31\%), whereas majority of the people who had never previously visited a dentist had education levels of 1 st to $7^{\text {th }}$ grade.

Majority of the people (83\%) who had never previously visited a dentist had an income of Indian rupees 500-3000 per month. Majority of the people $(38 \%)$ who had previously visited a dentist had an income of Indian rupees 3000-15000 per month.

Majority of the people who had previously visited a dentist said the time since their last dental visit was more than 2 years (38.5\%), followed by less than 6 months ago (31.4\%) (Table 1).

When the people who had previously visited a dentist were asked the reason for their last visit, the majority of them said they had visited because of dental pain (35.3\%), followed by decayed teeth $(27 \%)$. None of them said they had visited the dentist for routine oral examination (Table 2).

When the people were categorized according to the type of treatment received in their last dental visit, majority of the people said they had undergone extraction of teeth $(44.3 \%)$, followed by restoration of teeth (31.3\%) (Table 3).

Among people who had never previously visited a dentist, the reason most frequently cited for not visiting was the presence of a "dental problem which was not severe enough to go to a dentist" $(43.1 \%)$. This was followed by the reason that they "did not care" or they "neglected" their dental problems (22.4\%). The "cost of dental treatment" was a barrier for $6.9 \%$ of the people. The "fear of dentist/dental treatment" was a barrier for a very small number of people (1.7\%) (Table 4).

\section{DISCUSSION}

The study results showed that majority of the people visited a dentist more than 2 years ago, followed by less than 6 months ago. Similar results

Table 1. Distribution of previous visitors according to time since last dental visit.

\begin{tabular}{lc}
\hline Time since last dental visit & Total=122 \\
& $\mathrm{n}(\%)$ \\
\hline Less than 6 months & $38(31.4 \%)$ \\
6 months - 01 year & $18(14.6 \%)$ \\
01 year-02 years & $19(15.5 \%)$ \\
More than 2 years & $47(38.5 \%)$ \\
\hline
\end{tabular}

Table 2. Distribution of previous visitors according to reason for last dental visit.

\begin{tabular}{lc}
\hline Reason for last dental visit & $\mathrm{T}=122$ \\
& $\mathrm{n}(\%)$ \\
\hline Pain & $43(35.2 \%)$ \\
\hline Decayed teeth & $33(27.0 \%)$ \\
\hline Mobile teeth & $12(9.8 \%)$ \\
\hline Stains & $09(7.3 \%)$ \\
\hline Missing teeth & $02(1.6 \%)$ \\
\hline Trauma & $02(1.6 \%)$ \\
\hline Malocclusion & $02(1.6 \%)$ \\
\hline Bad breath & $01(0.8 \%)$ \\
\hline Combination of above & $18(14.7 \%)$ \\
\hline
\end{tabular}


have been reported in some other studies. ${ }^{4,12}$ Time since the last dental visit represents dental care that was initiated by the people and therefore could reflect personal motivation and independent decision making. Not visiting a dentist in the previous 2 years means that the people have poor preventive oral health practices, and they delay visiting a dentist until they have an acute dental problem, which forces them to make frequent visits thereafter. Though 6-monthly dental visits are recommended by most dentists, only a few individuals comply with this recommendation.

Pain was the predominant reason for visiting a dentist for the majority of people, followed by the decayed teeth. That the pain is the predominant reason for dental visits has also been shown in many other studies. 2,4,13,14,15 Dental pain adversely affects the quality of life, normal functioning, and daily living of people, and most dental visits are aimed at immediate relief of pain. Patients often present themselves for dental care at the later stages of dental disease when overt symptoms such as pain and extreme discomfort appear, rather than earlier, i.e., a problem-oriented visit rather than a prevention-oriented one.

None of the people interviewed said they had visited a dentist for routine oral examination. This shows that people do not believe in the value of regular dental visits, and they have very poor preventive practices.
During their previous dental visit, majority of the people had undergone extraction, followed by the restoration of teeth. This may indicate that patients present themselves for care at the later stage of dental disease, when the teeth cannot be salvaged, and the only option would be extraction. The extended delay may account for the high prevalence of patients undergoing extraction. The attitude of people may imply that people do not accept dental treatment as a viable alternative to decay and loss of teeth.

The most frequently cited reason for not visiting a dentist was the presence of a dental problem that was not severe enough to initiate a dental visit. This shows that people do not go to a dentist unless severe symptoms appear. Dentistry is perceived to be a useful service only when necessary but is not a crucial part of overall health. ${ }^{16}$ The belief that there was no need to visit a dentist unless pain was present was reported in a study done on the Kuwaiti adults. ${ }^{4}$

The neglect of dental problems was the second most commonly cited reason for not visiting a dentist. This shows the complacence towards one's oral health and the low priority given to oral problems as people think dental problems are not life threatening.

The lack of perceived need was the third most commonly reported reason for not visiting a dentist. Similar results have been reported in several

Table 3. Distribution of previous visitors according to treatment received in the last dental visit.

\begin{tabular}{|c|c|}
\hline Treatment received in the last dental visit & $\begin{array}{l}\mathrm{T}=122 \\
\mathrm{n}(\%)\end{array}$ \\
\hline Extraction of teeth & $54(44.3 \%)$ \\
\hline Restoration of teeth & $41(33.6 \%)$ \\
\hline Professional cleaning of teeth & $20(16.3 \%)$ \\
\hline Prosthetic treatment & $05(4.0 \%)$ \\
\hline Any other (Orthodontic treatment) & $02(1.6 \%)$ \\
\hline \multirow[t]{2}{*}{ Reason for not visiting a dentist } & $\mathrm{T}=58$ \\
\hline & $\mathrm{n}(\%)$ \\
\hline Problem not severe enough to go to a dentist & $25(43.1 \%)$ \\
\hline Do not care/ neglect oral problems & $13(22.4 \%)$ \\
\hline Have no dental problem & $09(15.5 \%)$ \\
\hline Visiting a dentist affects daily routine/work & $05(8.6 \%)$ \\
\hline Dental treatment is costly & $04(6.9 \%)$ \\
\hline Fear of dentist/ dental treatment & $01(1.7 \%)$ \\
\hline Dental treatment is lengthy/ Frequent visits are required & $01(1.7 \%)$ \\
\hline
\end{tabular}


other studies. 5, 10,17,18 The finding shows that inability to recognize the need for dental treatment in one's self acts as a barrier to utilization of dental services. Kiyak and Reichmuth have stated that the primary reason why people seek health services is their belief that they need health care and that the situation will get worse without professional help. ${ }^{18}$

The interference with daily routine or work was another reason cited by some people for not visiting a dentist. This finding is similar to that of a study reported from Malaysia. ${ }^{14} \mathrm{~A}$ visit to a dentist may lead to the loss of wages for people who are paid on a daily basis, and for salaried people, there may be difficulty in arranging working hours. Getting sick leave for dental problems may be difficult as they are not considered to be serious enough to take a leave.

Contrary to the results of many studies, the cost of treatment was cited as a barrier for using dental services only by $7 \%$ of people. ${ }^{5,14,16}$ As the study was conducted in a dental college hospital where the services are offered at reasonable fee when compared to private clinics, the cost was probably not seen as a major barrier.

One surprising finding of the study was that the fear of dentist or dental treatment was cited by a very few people as a reason for not visiting the dentist. This may be because of a possible Hawthorne effect, i.e., the mere fact that these people were reporting to a dentist might have made them to answer otherwise. Contrary to the finding in this study, the fear has been reported as a significant barrier for utilization of dental services in many studies. 4,10

One limitation of the study is that it was a selfreport survey. So, there is a possibility of response bias. Not controlling for socio-demographic variables as confounding factors for dental service use and non-inclusion of all potential barriers for dental service use are other limitations of the study. Since the study was hospital based and employed deliberate sampling, the results cannot be generalized at the community level.

The study, though it was carried out on a small scale, may provide an initial step in understanding which variables are important in utilization or non-utilization of dental care. But understanding treatment seeking behavior is a complex process. One small attempt over a limited period of time does not answer all questions. In future studies, it will be necessary to extend the analysis to a larger sample of individuals at the community level.

The findings of the study showed that the "pain" was the main reason for visiting a dentist. The major reason cited for not visiting a dentist was the presence of a dental problem that was not severe enough to go to a dentist. It can be concluded that the majority of people were "problem-oriented visitors" rather than "prevention-oriented visitors."

The findings of the study have important implications. As flag bearers of oral health, dental practitioners across locations must be willing not only to provide dental care to patients but also to educate them regarding the importance of regular dental care, discuss barriers for dental care, and promote attitudinal changes towards a positive health-seeking behavior. An active assessment of and attention to factors that facilitate and hinder dental service use is crucial while formulating oral health policies.

\section{ACKNOWLEDGEMENTS}

The authors sincerely acknowledge the co-operation of the people who participated in the study.

\section{REFERENCES}

1. Petersen PE. Global policy for improvement of oral health in the 21st century -implications to oral health research of World Health Assembly 2007, World Health Organization. Community Dent Oral Epidemiol 2009;37:1-8.

2. Cohen LA, Bonito AJ, Eicheldinger C, Manski RJ, Masek MD, Edwards RR et al. Behavioral and socioeconomic correlates of dental problem experience and patterns of health care-seeking. J Am Dent Assoc 2011;142:137-149.

3. Arora SA, Jayna A, Sharma A and Atri M. Socio-demographic factors influencing preventive dental behaviors in an adult dentate population: a questionnaire based study. Indian J of Dental Advancements 2011;3:483-491.

4. Al-Shammari KF, Al-Ansari JM, Al-Khabbaz AK and Honkala S. Barriers to seeking preventive dental care by Kuwaiti adults. Med Princ Pract 2007;16:413-419.

5. Pedersen PH, Vigild M, Nitschke I and Berkey DB. Dental care for aging populations in Denmark, Sweden, Norway, United Kingdom and Germany. J Dent Educ 2005;69:987997. 
6. Stewart DC, Ortega AN, Dausey D and Rosenheck R. Oral health and use of dental services among Hispanics. J Public Health Dent 2002;62:84-91.

7. Formicola AJ, Stavisky J and Lewy R. Cultural competency: dentistry and medicine learning from one another. $J$ Dent Educ 2003;67:869-875.

8. Bachrach CA and Abeles RP. Social science and health research: growth at the National Institutes of Health. Am J Pub Health 2004;94:22-28.

9. Atchison KA and Dubin LF. Understanding health behavior and perceptions. Dent Clin North Am 2003;47:21-39.

10. Poudyal S, Rao A, Shenoy R and Priya H. Utilization of dental services in a field practice area in Mangalore, Karnataka. Indian J Community Med 2010;35:424-425.

11. Cohen LK. Converting unmet need for care to effective demand. Int Dent $J$ 1987;37:114-116.

12. Manski RJ and Magder LS. Demographic and socioeconomic predictors of dental care utilization. $J$ Am Dent Assoc 1998;46:432-439.

13. Ekanayake L and Mendis R. Self-reported use of dental services among employed adults in Sri Lanka. Int Dent $J$ 2002;19:151-156.

14. Jaafar N, Jalalludin RL, Razak IA and Esa R. Investigation of delay in utilization of government dental services in Malaysia. Community Dent Oral Epidemiol 1992;20:144-147.

15. Kuthy RA, Odom JG, Salsberry PJ, Nickel JL and Polivka BJ. Dental utilization by low-income mothers. $J$ Public Health Dent 1998;58:44-50.

16. Heaton LJ, Smith TA and Raybould TP. Factors influencing use of dental services in rural and urban communities: considerations for practitioners in underserved areas. $J$ Dent Educ 2004;68:1081-1089.

17. Kiyak HA. Reducing barriers to older persons' use of dental services. Int Dent J 1989;39:95-102.

18. Kiyak HA and Reichmuth M. Barriers to and enablers of older adults' use of dental services. J Dent Educ 2005;69:975986. 